\title{
miRNA interaction with 5'UTR, CDS, 3'UTR mRNA candidate genes of breast cancer subtypes
}

\author{
D. Aisina*, R. Niyazova, Sh. Atambayeva, A. Ivashchenko \\ al-Farabi Kazakh National University, Almaty, Kazakhstan \\ *e-mail:dana.aisina03@gmail.com
}

Key words: breast cancer, subtypes, genes, miRNAs

Breast cancer subtypes are distinguished by a set of candidate genes involved in the development of this disease. The expression of many genes is regulated by binding of their mRNAs with miRNAs. It is required to identify which candidate genes can interact with miRNAs. The MirTarget program defines the following features of binding: start of the initiation of miRNA binding to mRNAs; localization of miRNA binding sites in 5'UTRs, CDSs and 3'UTRs; free energy of binding; schemes of nucleotide interactions between miRNAs and mRNAs. mRNAs of many genes have miRNA binding sites with overlapping nucleotide sequences (clusters) located in 5'UTR, CDS, 3'UTR. There are cluster of three sites of different miRNAs in the 5'UTR mRNA EPOR, MAZ and NISCH candidate genes (her2 subtype), cluster of 11 sites in the CDS mRNA MAZ gene, clusters of three sites and 17 sites in the 3'UTR mRNA BRCA2 gene and CDK6 genes, respectively. Candidate genes of the triple-negative subtype are targets: in the 5'UTR mRNA CBL gene are 11 sites, mRNA MMP2 gene - five sites, mRNA RAB5A gene are two cluster each of three sites, in the 3'UTR mRNA $S F N$ gene - 18 sites. Candidate genes of luminal A and B subtypes are targets: in the 5'UTR mRNA FOXA1 gene are 19 sites, mRNA HMGA2 gene - 12 sites, mRNA TGFB1 gene - two clusters of three and four sites. There are clusters of four sites and three sites in the CDS mRNA ITGB1 and SOX4 genes, respectively; clusters of three sites, four sites and five sites in the 3'UTR mRNA $S M A D 3, S O X 4$ and GFB1 genes, respectively. The organization of binding sites into clusters several times reduces the proportion of binding sites in nucleotides in $5^{\prime} \mathrm{UTR}$, CDS and 3'UTR. Based on the results, associations of miRNAs and mRNAs candidate genes are recommended for developing methods of breast cancer subtypes diagnostics. 